\title{
Effective properties of fibers with various ratios of phase stiffness
}

\author{
P. Procházka \\ CTU Prague, Civil Engineering, Prague, Czech Republic
}

\begin{abstract}
In the paper a new procedure for effective properties of nonlinear composites will be proposed. Based on boundary element method various ratios of phase stiffnesses are studied. Special properties of the stress distribution (or concentration factors) on a unit cell are utilized. The overall properties start with Hashin-Shtrikman idea, which is transformed to a numerical framework. Without loss of generality geometrical boundary conditions on the boundary of a unit cell are prescribed in this study. A special case of the presented approach is the effect of pore pressure, occurring in fiber reinforced concrete, for example. Also nonlinear behavior of the phases is taken into consideration, especially the von Mises-Huber-Hencky criterion is adopted in the model of matrix. The examples are exclusively prepared for two-phase materials.
\end{abstract}

Keywords: boundary element method, various stiffness of phases, plasticity, Hashin-Shtrikman variational principle.

\section{Introduction}

Eigenstresses and eigenstrains play a very important role in many branches of applied mechanics, e.g., in composites, geotechnics, concrete structures, etc. In previous papers, the authors have formulated an effective approach to the analysis and optimization of inhomogeneous bodies with prescribed boundary displacements or tractions and have used the transformation field analysis for relating the components of stress or strain tensors and of eigenstrains or eigenstresses. The transformation field analysis established by Dvorak and Procházka in [1] has been applied to localization of stresses and strains in twophase composites. The eigenstresses stood for relaxation stresses while eigenstrains represented plastic strains. This idea was extended in [2], where 
applications of a large scale of combinations of internal material situations using boundary element method with eigenstress of composite structures were considered.

In [3] an attempt for obtaining effective material properties of a nonlinear isotropic composite has been made, using the basic ideas of Hashin and Shtrikman, [4]. A new variational approach was proposed that provides the effective energy potentials of nonlinear composites in terms of the corresponding energy potentials for linear composites with the same microstructural distributions.

Our approach is based on the idea of extended Hashin-Shtrikman variational principles for nonhomogeneous bodies. By means of internal parameters, eigenstrains or eigenstresses, involved in H-S principles, it is possible to obtain new bounds on mechanical properties of the trial material, increase the bearing capacity of structures, and to minimize the stress excesses.

\section{Basic relations}

We start with basic relations which are valid in mechanics of continuum and are appropriate for our next considerations. Denote by $\Omega$ a bounded domain, $\Gamma \equiv \Gamma_{u} \cup \Gamma_{p}, \Gamma_{u} \cap \Gamma_{p}=0$, being its Lipschitz's boundary, both representing the trial body. On $\Gamma_{u}$ the displacement vector $\boldsymbol{u}=\left\{u_{1}, u_{2}, u_{3}\right\}$ is prescribed, and on $\Gamma_{p}$ the vector of tractions $\boldsymbol{p}=\left\{p_{1}, p_{2}, p_{3}\right\}$ is given. Recall the relation stresses-tractions on the boundary $\Gamma_{p}: p_{i}=\sigma_{i j} n_{j}$, where $\boldsymbol{n} \equiv\left\{n_{1}, n_{2}, n_{3}\right\}$ is the outward unit normal to the boundary, $\sigma_{i j}, \varepsilon_{i j}$ are components of stress and strain tensors, respectively. form

Hooke's law for anisotropic and inhomogeneous field is introduced in the

$$
\sigma_{i j}(\boldsymbol{x})=L_{i j k l}(\boldsymbol{x}) \varepsilon_{k l}(\boldsymbol{x})+\lambda_{i j}(\boldsymbol{x}), \varepsilon_{i j}(\boldsymbol{x})=M_{i j k l}(\boldsymbol{x}) \sigma_{k l}(\boldsymbol{x})+\mu_{i j}(\boldsymbol{x})
$$

where $\lambda_{i j}$ are components of the eigenstress tensor, $\mu_{i j}$ are the components of the eigenstrain tensor, $\boldsymbol{x}$ is a position at which the material relations are studied, $L_{i j k l}$ are components of the material stiffness tensor and $M_{i j k l}$ describe its compliance material tensor, both with the standard symmetry; the subscripts $i, j, k, l$ run the set $\{1,2,3\}$,

Moreover, we have

$$
L_{i j k l} M_{k l m n}=I_{i j m n}, \quad I_{i j m n}=\frac{1}{2}\left(\delta_{i m} \delta_{j n}+\delta_{i n} \delta_{j m}\right)
$$

where $I_{i j m n}$ characterises the fourth-order unit tensor, $\delta_{i j}$ is the Kronecker delta being equal to one for $i=j$ and zero otherwise.

Note that for a homogeneous and isotropic material the material stiffness matrix has the form 


$$
L_{i j k l}=\lambda \delta_{i j} \delta_{k l}+2 \mu I_{i j k l}
$$

where $\lambda$ and $\mu$ are Lame's constants. Instead of $\mu$, the shear modulus $G$ is sometimes introduced.

Comparing both equations (1), we get

$$
\lambda_{i j}=-L_{i j k l} \mu_{k l}, \quad \mu_{i j}=-M_{i j k l} \lambda_{k l}
$$

Kinematic equations may be written as

$$
\varepsilon_{i j}=\frac{1}{2}\left(\frac{\partial u_{i}}{\partial x_{j}}+\frac{\partial u_{j}}{\partial x_{i}}\right)
$$

Note that displacements are said to be kinematically admissible if the relation (5) holds.

Eventually, static equations or equations of equilibrium yield

$$
\frac{\partial \sigma_{i j}}{\partial x_{j}}=0
$$

provided no volume weight forces are taken into account. The last relation has to be taken in the sense of distributions.

Note that one says that the stress tensor is statically admissible or its components are statically admissible, if statistical boundary conditions on $\Gamma_{p}$ are prescribed and (6) is fulfilled.

Substituting the kinematical equations into the equations of equilibrium leads to Lame's equations for the unknown displacement vector $\boldsymbol{u}$, which are written in the sense of distributions:

$$
\frac{\partial}{\partial x_{j}}\left[L_{i j k l}\left(\frac{\partial u_{k}}{\partial x_{l}}+\frac{\partial u_{l}}{\partial x_{k}}-2 \mu_{k l}\right)\right]=0 \quad \text { in } \Omega,
$$

or alternatively

$$
\frac{\partial}{\partial x_{j}}\left[L_{i j k l}\left(\frac{\partial u_{k}}{\partial x_{l}}+\frac{\partial u_{l}}{\partial x_{k}}-2 \lambda_{k l}\right)\right]=0 \quad \text { in } \Omega
$$

for a given field $\mu_{k l}$, or $\lambda_{k l}$.

Recall that on the part $\Gamma_{u}$ of the boundary $\Gamma$ the displacement vector $\boldsymbol{u}=\overline{\boldsymbol{u}}$ is prescribed, and the traction field $\boldsymbol{p}=\overline{\boldsymbol{p}}$ is given on $\Gamma_{p}$.

\section{Localization using the BEM}

In this section we use extend Hashin-Shtrikman variational principle [4], by introducing both the eigenstrain and eigenstress fields into the formulation. For the sake of simplicity assume that no body forces are present. 
The idea of Hashin and Shtrikman consists in introducing new variables $\tau_{i j}$ (components of the polarization tensor). Let us consider again a bounded domain $\Omega$ with its bounded Lipschitz's boundary $\Gamma$ and with subdomains $\Omega_{i}, i=$ $1, \ldots, n$, describing local inhomogeneities.

The overall strain $E_{i j}$ is assumed to be given independently of the shape of the unit cell and of the shape of the fiber. The loading of this unit cell will be given by unit impulses of $E_{i j}$, i.e. we successively select $E_{i_{0} j_{0}}=E_{j_{0} i_{0}}=1 ; E_{i j}=0$ for either $i_{0} \neq i$ or $j_{0} \neq j$. How to select the unit impulses of the overall strain components will be discussed later on.

Now we concentrate our attention on the approach of computing the concentration factors, which play the most important role in our solution of the optimal problem. The idea is adapted to the computation of the concentration factors. Note that approximate estimates of the concentration factors can be done using several formulas (Mori-Tanaka, self/consistent method, etc.). We are going to show in this paper relatively simple calculation of the factors using the boundary elements.

First let us specify the boundary conditions, being equivalent to the unit impulses of the overall strain components. In elasticity it is possible to prescribe the overall strain field all over the domain $\Omega$ of the unit cell. Then the solution of responses to the unit impulses is given by the periodicity conditions and tractions along the interfacial surface $\Gamma$ between fibers and matrix. This approach is little bit cumbersome in case of debonding is admitted. The latter case is not considered here, but we apply more general form of introducing the unit impulses. It is well known that because of identity; see $\left(2_{3}\right)$ and Green's theorem:

$$
\begin{aligned}
E_{i j}=\int_{\Omega} \varepsilon_{i j}(\boldsymbol{y}) \mathrm{d} \Omega=\frac{1}{2} \int_{\Omega}[ & \left.\frac{\partial u_{i}}{\partial y_{j}}(\boldsymbol{y})+\frac{\partial u_{j}}{\partial y_{i}}(\boldsymbol{y})\right] \mathrm{d} \Omega= \\
& =-\frac{1}{2} \int_{\partial \Omega}\left[u_{i}(\boldsymbol{y}) n_{j}(\boldsymbol{y})+u_{j}(\boldsymbol{y}) n_{i}(\boldsymbol{y})\right] \mathrm{d} \gamma(\boldsymbol{y})
\end{aligned}
$$

From (9) it immediately follows that the unit responses are given by prescribed displacements along the boundary of the unit cell.

The procedure is split into two steps. Assume the above described surface displacements to be prescribed along the entire boundary $\partial \Omega$ and there are no body forces here. In the first step, the unit cell obeys static equilibrium equations and linear homogeneous Hooke's law (homogeneous and isotropic medium):

$$
\sigma_{i j}^{0}=L_{i j k l}^{0} \varepsilon_{k l}^{0}, \text { in } \Omega, \quad \text { given boundary conditions are fulfilled on } \partial \Omega .
$$

$L_{i j k l}^{0}$ are components of a not yet determined material stiffness matrix (stiffness tensor). These components will be stated later. Such a medium is called comparative one. 
The solution of (10) is easy, as the comparative medium is homogeneous and isotropic:

$$
u_{i}^{0}=E_{i j} y_{j}, \quad \varepsilon_{i j}^{0}=E_{i j} \quad \text { in } \Omega, \quad p_{i}^{0}=\sigma_{i j}^{0} n_{j}=L_{i j k l}^{0} E_{k l} n_{j} \quad \text { on } \partial \Omega
$$

In the second step a geometrically identical unit cell is considered. Also the loading and boundary conditions on $\partial \Omega$ remain valid. Define

$$
\begin{aligned}
& \bar{u}_{i}=u_{i}-u_{i}^{0}=u_{i}-E_{i j} y_{j}, \bar{\varepsilon}_{i j}=\varepsilon_{i j}-\varepsilon_{i j}^{0}=\varepsilon_{i j}-E_{i j}, \\
& \bar{\sigma}_{i j}=\sigma_{i j}-\sigma_{i j}^{0}=\sigma_{i j}-L_{i j k l}^{0} E_{k l} \text { in } \Omega \quad \bar{u}_{i}=0 \text { on } \Gamma_{u}, \bar{p}_{i}=0 \text { on } \Gamma_{p}
\end{aligned}
$$

Our next aim is to determine primed quantities, components of displacement vector $\bar{u}_{i}$ and components of strain and stress tensors $\bar{\varepsilon}_{i j}$ and $\bar{\sigma}_{i j}$. In order to do so, system of fifteen equations of elasticity (2) has to be formulated for the primed set. We start with Hooke's law, which is valid for heterogeneous medium:

$$
\sigma_{i j}(y)=L_{i j k l}(y) \varepsilon_{k l}(y) \quad \text { in } \Omega
$$

Since the material stiffness tensor appears to be nonhomogeneous and unisotropic, idea used in [4], among others, will be adapted also here:

$$
\sigma_{i j}(\boldsymbol{y})=L_{i j k l}^{0} \varepsilon_{k l}(\boldsymbol{y})+\tau_{i j}(\boldsymbol{y}) \quad \text { in } \Omega
$$

where $\tau_{i j}$ are components of the polarization tensor and the direct relation between stresses and strains becomes homogeneous and isotropic, so that integral formulation of elastic problem may be formulated. Subtracting (13) and (12) yields:

$$
\tau_{i j}=\left[L_{i j k l}\right] \varepsilon_{k l}, \quad\left[L_{i j k l}\right]=L_{i j k l}-L_{i j k l}^{0}
$$

which can be considered a definition of polarization tensor. Moreover, transformation to the primed system will not disturb the direct relation stresses strains, as after substituting $(13)$ to $\left(11_{3}\right)$ gives:

$$
\bar{\sigma}_{i j}=\sigma_{i j}-\sigma_{i j}^{0}=L_{i j k l}^{0} \varepsilon_{k l}+\tau_{i j}-\sigma_{i j}^{0}=L_{i j k l}^{0} \varepsilon_{k l}+\tau_{i j}-L_{i j k l}^{0} E_{k l}=L_{i j k l}^{0} \bar{\varepsilon}_{k l}+\tau_{i j}
$$

Since both $\sigma_{i j}$ and $\sigma_{\mathrm{i} j}^{0}$ are statically admissible, it holds (the following equations must be defined in the sense of distributions):

$$
\frac{\partial\left(L_{i j k l}^{0} \bar{\varepsilon}_{k l}+\tau_{i j}\right)}{\partial y_{j}}=0 \quad \text { in } \Omega, \quad \bar{u}_{i}=u_{i}-u_{i}^{0}, \quad \bar{p}_{i}=p_{i}-p_{i}^{0} \quad \text { on } \partial \Omega
$$

Following the assumption of the same prescribed boundary conditions, some of the terms in (16) disappear. 
Owing to constant distribution of $L_{i j k l}^{0}$ in $\Omega$, the equivalent integral formulation can be written as:

$$
\begin{aligned}
& \bar{u}_{m}(\boldsymbol{\xi})= \int_{\partial \Omega} p_{m i}^{*}(\boldsymbol{y}, \boldsymbol{\xi}) \bar{u}_{i}(\boldsymbol{y}) \mathrm{d} \gamma(\boldsymbol{y})-\int_{\partial \Omega} u_{m i}^{*}(\boldsymbol{y}, \boldsymbol{\xi}) \bar{p}_{i}(\boldsymbol{y}) \mathrm{d} \gamma(\boldsymbol{y})+ \\
&+\left(\left[L_{i j k l}^{\mathrm{f}}-L_{i j k l}^{0}\right] \int_{\Omega^{\mathrm{f}}}+\left[L_{i j k l}^{\mathrm{m}}-L_{i j k l}^{0}\right] \int_{\Omega^{\mathrm{m}}}\right)_{\varepsilon_{m i j}}^{*}(\boldsymbol{y}, \boldsymbol{\xi}) \bar{\varepsilon}_{k}(\boldsymbol{y}) \mathrm{d} \Omega \\
& c_{m n}(\boldsymbol{\xi}) \bar{u}_{n}(\boldsymbol{\xi})=\int_{\partial \Omega} p_{m i}^{*}(\boldsymbol{y}, \boldsymbol{\xi}) \bar{u}_{i}(\boldsymbol{y}) \mathrm{d} \gamma(\boldsymbol{y})-\int_{\partial \Omega} u_{m i}^{*}(\boldsymbol{y}, \boldsymbol{\xi}) \bar{p}_{i}(\boldsymbol{y}) \mathrm{d} \gamma(\boldsymbol{y})+ \\
&+\left(\left[L_{i j k l}^{\mathrm{f}}-L_{i j k l}^{0}\right] \int_{\Omega^{\mathrm{f}}}+\left[L_{i j k l}^{\mathrm{m}}-L_{i j k l}^{0}\right] \int_{\Omega^{\mathrm{m}}} \sigma_{m i j}^{*}(\boldsymbol{y}, \boldsymbol{\xi}) \bar{\varepsilon}_{k}(\boldsymbol{y}) \mathrm{d} \Omega \quad \boldsymbol{\xi} \in \partial \Omega\right.
\end{aligned}
$$

where $c_{m n}$ are components of a tensor depending on position $\xi \in \partial \Omega$ and the quantities with asterisks are given kernels.

Differentiating (17) by $\xi_{n}$ and putting $\xi \in \partial \Omega$ provides

$$
\begin{aligned}
& \bar{\varepsilon}_{m n}(\boldsymbol{\xi})=\int_{\partial \Omega} P_{m i}^{*}(\boldsymbol{y}, \boldsymbol{\xi}) \bar{u}_{i}(\boldsymbol{y}) \mathrm{d} \gamma(\boldsymbol{y})-\int_{\partial \Omega} U_{m i}^{*}(\boldsymbol{y}, \boldsymbol{\xi}) \bar{p}_{i}(\boldsymbol{y}) \mathrm{d} \gamma(\boldsymbol{y})+ \\
& +\left(\left[L_{i j k l}^{\mathrm{f}}-L_{i j k l}^{0}\right] \int_{\Omega^{\mathrm{f}}}+\left[L_{i j k l}^{\mathrm{m}}-L_{i j k l}^{0}\right] \int_{\Omega^{\mathrm{m}}} \sum_{m i j}^{*}(\boldsymbol{y}, \boldsymbol{\xi}) \bar{\varepsilon}_{k}(\boldsymbol{y}) \mathrm{d} \Omega+\right.\text { convected term }
\end{aligned}
$$

First, let $L_{i j k l}^{0} \equiv L_{i j k l}^{\mathrm{f}}$. Eliminating unknown boundary values from (18) and (19) we obtain the relation

$$
\varepsilon_{i j}^{\mathrm{m}}(\overline{\boldsymbol{u}}(\boldsymbol{y}))=\beta_{i j k l}^{\mathrm{m}}(\boldsymbol{y}) E_{k l}
$$

and if $L_{i j k l}^{0} \equiv L_{i j k l}^{\mathrm{m}}$ then

$$
\varepsilon_{i j}^{\mathrm{f}}(\overline{\boldsymbol{u}}(\boldsymbol{y}))=\beta_{i j k l}^{\mathrm{f}}(\boldsymbol{y}) E_{k l}
$$

This process leads to a fourth-order "concentration factor tensor" $A_{i j k l}$ defined as

$$
\varepsilon_{i j}^{p}(\boldsymbol{u}(\boldsymbol{y}))=\left(I_{i j k l}+\beta_{i j k l}^{p}(\boldsymbol{y})\right) E_{k l}=A_{i j k l}^{p}(\boldsymbol{y}) E_{k l}
$$

where the superscript $p \equiv \mathrm{f}$ for $\boldsymbol{y} \in \Omega^{\mathrm{f}}$ and $p \equiv \mathrm{m}$ for $\boldsymbol{y} \in \Omega^{\mathrm{m}}$.

Since it obviously holds

$$
<A_{i j k l}^{\mathrm{f}}>_{\mathrm{f}}+<A_{i j k l}^{\mathrm{m}}>_{\mathrm{m}}=1
$$


one does not need to compute both concentration factors. It is sufficient to draw a concentration on fiber, when dealing with concrete composites (the fiber ratio is very small), or on matrix, if the matrix volume ratio is large and the material behavior of stresses on matrix is nearly uniformly distributed.

\section{Inelastic behavior of one phase}

Assume that in the domain of elastic stresses a set

$$
F\left(\sigma_{i j}\right)<0
$$

Is given and the domain of the plastic surface is defined by the equality:

$$
F\left(\sigma_{i j}\right)=0
$$

Here $\sigma_{i j}$ are components of the symmetric stress tensor. Function $F$ is convex and continuous with respect to the arguments $\sigma_{i j}$ as in $\mathfrak{R}_{6}$ the admissible set $F\left(\sigma_{i j}\right) \leq 0$ is closed convex and $F\left(\sigma_{i j}\right)$ involves only deviatoric stresses $\sigma_{i j}^{\mathrm{D}}=\sigma_{i j}-\frac{\delta_{i j}}{3}\left(\sigma_{k k}\right)$, so that the deviatoric stress tensor possesses zero trace. Namely, for the von Mises-Huber-Hencky it holds:

$$
F\left(\sigma_{i j}\right)=\frac{1}{2} \sigma_{i j}^{\mathrm{D}} \sigma_{i j}^{\mathrm{D}}-k^{2}
$$

where $k$ is another material constant. Formulating a set

$$
K=\left\{\boldsymbol{\sigma} ; \quad \boldsymbol{\sigma}=\left\{\sigma_{i j}\right\} \in \mathfrak{R}_{6}, \quad \sigma_{i j}=\sigma_{j i}, \quad F\left(\sigma_{i j}\right) \leq 0\right\}
$$

the constitutive equations are written as:

$$
\varepsilon_{i j}(\dot{\boldsymbol{u}})=M_{i j k l} \dot{\sigma}_{k l}+\lambda_{i j}
$$

where $\varepsilon_{i j}$ are components of the strain tensor, $M_{i j k l}$ is the compliance tensor of elastic parameters, overdot means time derivative (response of and increment of loading), $\lambda_{i j}$ are components of the eigenstress tensor, here standing for components of the relaxation stress being defined as:

$$
\lambda_{i j}= \begin{cases}0, & \text { if } F(\boldsymbol{\sigma})<0 \\ \frac{1}{2 \mu}\left[\sigma_{i j}-\left(P_{K} \boldsymbol{\sigma}\right)_{i j}\right] & \text { if } F(\boldsymbol{\sigma}) \geq 0\end{cases}
$$

and a mapping $\sigma \rightarrow P_{K}(\sigma)$ is an orthogonal projection in the domain of Euclidean structure $\sigma \in \mathfrak{R}_{6}$ on a convex set $K$. Coefficient $\mu$ is possible to consider as the coefficient of viscosity in describing fluid movement and is identified as shear modulus $G$ in solid materials. If only constant $\sigma_{i j}$ appear in the development of material stages, i.e. $\dot{\sigma}_{i j}=0$, the latter equations turn to 
$\sigma_{i j}=\left(P_{K} \boldsymbol{\sigma}\right)_{i j}+2 \mu \varepsilon_{i j}(\dot{\boldsymbol{u}})$ and for fluids their movement is described by the Navier-Stokes equations it holds:

$$
\sigma_{i j}=-p \delta_{i j}+2 \mu \varepsilon_{i j}(\dot{\boldsymbol{u}})
$$

In case Mises condition is allowed for the relaxation stresses are derived as:

$$
\lambda_{i j}= \begin{cases}0, & \text { if } F(\sigma)<0 \\ \frac{1}{2 \mu} \frac{\sqrt{\sigma_{\mathrm{II}}}-k}{\sqrt{\sigma_{\mathrm{II}}}} \sigma_{i j}^{\mathrm{D}} & \text { if } F(\sigma) \geq 0\end{cases}
$$

where $\sigma_{\mathrm{II}}=\frac{1}{2} \sigma_{i j}^{\mathrm{D}}$.

\section{Conclusions}

In this paper combustion of the concrete lining and the rock surrounding the tunnel is solved by Smooth Particle Hydrodynamics Method. The results are partly compared with experiments on concrete slabs burned in a furnace. Particularly, influences of fibers from fused basalt are observed and the composite with concrete matrix is evaluated after carrying out tests in Innsbruck University. The basalt material seems to be quite suitable for this case of endangered concrete linings. The reason is that at lower temperatures it behaves as stiffener of the concrete matrix and overcoming the temperature of $1000^{\circ} \mathrm{C}$ it becomes melting and enables vapor to escape from the concrete not to cause any larger damage. Consequently, in comparison to other types of fibers fused basalt serves in a proper way. Natural fibers are very suitable for high temperatures (they burn out and the vapor can freely dilute in the air) and stiff fibers like steel serve a good reinforcement but too troublesome in he case of influence of high temperature.

The SPH method is perfectly suitable for solving such a problem. This method shows very promising time consumption of computer (it is basically low in comparing it with other numerical methods), but generally it suffers from one unpleasant property: inhomogeneous geometrical boundary conditions can be respected in the calculus in complicated way. In our case this type of boundary conditions does not play any decisive role. The boundary conditions are declared by time changing source of heat, and the damage in the material is calculated at the time-stage.

\section{Acknowledgement}

Work on this project has been financially supported by GACR, project No. $103 / 07 / 0304$. Sponsorship of CIDEAS is also acknowledged. 


\section{References}

[1] Dvorak, G.J., Procházka, P.P. Thin-walled composite cylinders with optimal fiber prestress. Composites Part B, 27B, 1996, 643-649

[2] Procházka, P.P., Sejnoha, J. Behavior of composites on bounded domain. BE Communications 7, 1996, 6-8

[3] Procházka, P.P., Sejnoha, J. Extended Hashin-Shtrikman variational principles. Applications of Mathematics, No. 4, 49, 2004, 357-372

[4] Hashin, Z., Shtrikman, S. A variational approach to the theory of the elastic behaviour of polycristals. J. Mech. Phys. Solids 10, 1962, 343-352

[5] Duvant, G., Lions, J-L. Les inéquations en méchanique et en physique. DUNOD, Paris 1972.

[6] Suquet, P.M. Elements of homogenization for inelastic solid mechanics. Lecture Notes in Physics 272: Homogenization Techniques for Composite Media, Eds E. Sanches.Palencia and A. Zaoui, Springer-Verlag 1987. 